\title{
Propagation Characteristics of Optical Long Period Fiber Gratings using Graphical Solution Methods
}

Felipe S. Delgado, Marco A. Jucá, Thiago V. N. Coelho, Alexandre B. dos Santos, Andres P. L. Barbero, Vinicius N. H. Silva

\begin{abstract}
In this paper, we explicitly show a complete analysis and detailed calculation of propagation characteristics of the effective refractive indices of the core and cladding for a long period grating employing both the Two-Layer and Three-Layer Geometries. The results are achieved employing graphical solution methods and presented in terms of the effective refractive indices of the core and cladding. Then, we compare the results with OptiGrating (Optiwave Systems Inc., Ottawa, Canada). Finally, we point the best model to evaluate the effective refractive indices, avoiding incorrect designs of the long period gratings.

Index Terms - Effective Refractive Index, Long Period Gratings, Propagation modes, cladding modes.
\end{abstract}

\section{INTRODUCTION}

Nowadays, long period gratings (LPGs) has a growing importance in the fields of optical communications and fiber sensors technologies [1-4]. In single mode fibers, LPGs couple energy from the fundamental guided mode propagating in the core $\left(L P_{01}\right)$ to different co-propagating symmetric cladding modes $\left(L P_{0 m}, m=2,3,4 \ldots\right)$, satisfying the phase-matching equation [5]:

$$
\Delta \beta^{(m)}=\beta_{c o}-\beta_{c l a d}^{(m)}=\frac{2 \pi}{\Lambda}
$$

where $\Delta \beta^{(m)}$ is the differential propagation constant, $\Lambda$ is the grating period, $\beta_{c o}$ and $\beta_{\text {clad }}^{(m)}$ are the propagation constants for the fundamental and mth cladding modes, respectively.

These cladding modes have different propagation characteristics that are dependent upon the host fiber and the refractive index of the external medium [6]. Also, they attenuate rapidly, due to large scattering losses at the cladding-air interface and bends in the fiber [7], and thus leave several attenuation bands centered at discrete resonant wavelengths $\left(\lambda_{\text {res }}^{m}\right)$ in the transmission spectrum, obtained through the phase-matching condition and represented as [6]:

$$
\lambda_{\text {res }}^{m}=\left(n_{e f f, c o}^{01}-n_{e f f, c l a d}^{m}\right) \Lambda
$$

where $n_{\text {eff,co }}^{01}$ is the effective refractive index of the fundamental core mode, $n_{e f f, c l a d}^{m}$ is the effective refractive index of the $m$ th cladding mode, and $\Lambda$ is the period of the LPG.

In order to estimate the effective refractive indices of a LPG, the propagation constants of the fundamental and cladding modes must be prior calculated. An analysis of the theoretical background of LPGs has revealed two main theoretical formulations for this purpose, both are based on weakly 
guided approximation [8] to determine the effective refractive index of the fundamental core mode. However, these theories distinguish in the analysis of the propagation characteristics of the cladding modes.

The first method, reported by Vengsarkar [9], is the Two-Layer Geometry model, which simplifies the analysis and calculation of the propagation characteristics of the cladding modes by ignoring the effect of the core. The second one, proposed by Erdogan [10-11], is the Three-Layer Geometry model. In this case, the propagation of the cladding modes are described more accurately since both the corecladding and cladding-ambient interfaces are taken into consideration to calculate the propagation constants and effective indices of the modes.

Reference [12] shows a complete method to determine the total transmission characteristics of LPG grating. The model treats interaction between the fundamental LP01 mode and high-azimutal-order cladding modes with arbitrary azimuthal and radial refractive index variations. This method is an excellent choice for the complete analysis of LPGs with different profiles but the graphical method presents less computational costs and can be used for simple LPG geometry applications.

This paper reflects a complete and detailed simulation analysis regarding the Two-Layer and ThreeLayer Geometries using a graphical solution method to estimate the propagation constants and the effective refractive indices of the LPG. We use the MATLAB (The MathWorks, Natick, Massachusetts, USA) to develop simulation processes aiming accurate results of the LPG parameters calculated. Also, we validate the results obtained by comparing them with OptiGrating (Optiwave Systems Inc., Ottawa, Canada). Finally, we define which geometry model will provide correct results, and thus, avoid erroneous design of the LPGs characteristics.

\section{DETERMINING THE EFFECTIVE REFRACTIVE INDEX OF THE CORE}

In order to evaluate the propagation characteristics of the guided core mode, optical fiber waveguide geometry is employed [13]. We assumed that the fiber geometry consists of two concentric cylinders with a step index profile that presents a uniform central core surrounded by an infinite and homogenous cladding [13-14]. Besides, we consider that the optical fiber exhibits a small normalized core-cladding index difference, so that the fiber can be assumed to be only weakly guiding $(\Delta \ll 1)$ and the linearly polarized $(L P)$ approximation [8] can be used to describe a mode guided by the fiber core.

Eventually, it is possible to find the propagation constant of the fundamental core mode $\left(L P_{01}\right)$ from a particular dispersion relation given by an eigenvalue equation, as follows [13-14]:

$$
u_{c o}\left(\frac{J_{1}\left(u_{c o}\right)}{J_{0}\left(u_{c o}\right)}\right)=w_{c o}\left(\frac{K_{1}\left(w_{c o}\right)}{K_{0}\left(w_{c o}\right)}\right)
$$

where $\mathrm{J}_{0}$ and $\mathrm{J}_{1}$ are Bessel functions of the first kind, of order zero and one, respectively, and $\mathrm{K}_{0}$ and $\mathrm{K}_{1}$ represent the modified Bessel functions of second kind, of order zero and one, respectively. Finally, $\mathrm{u}_{\mathrm{co}}$ and $\mathrm{w}_{\mathrm{co}}$ are the normalized transverse wave numbers described in terms of the normalized 
frequency of the fiber $(V)$, which is dependent upon the guide and light frequency, and defined as [15]:

$$
\begin{aligned}
& V=\left(\frac{2 \pi a_{c o}}{\lambda}\right) \sqrt{n_{c o}^{2}-n_{c l a d}^{2}} \\
& u_{c o}=\sqrt{V^{2}-w_{c o}^{2}}
\end{aligned}
$$

We can define the desired parameters of the optical fiber such as core diameter $\left(a_{c o}\right)$, and refractive index of the core $\left(n_{c o}\right)$ and cladding $\left(n_{\text {clad }}\right)$, that permit us to calculate the normalized frequency and then, we can use (5) to establish eigenvalues that satisfy (3).

Thus, we use a graphical solution method and plot the left-hand and right-hand sides of (3) on the same axis to obtain graphically the intersection point between them for the fundamental core mode $\left(L P_{01}\right)$. The coordinates at the point of intersection specify the eigenvalue and thus the normalized transverse wave numbers $\mathrm{u}_{\mathrm{co}}$ and $\mathrm{w}_{\mathrm{co}}$, these parameters are related to the propagation constant of the fundamental core mode $\left(\beta_{c o}\right)$, written as [16]:

$$
\beta_{c o}=\sqrt{\left(k n_{c o}\right)^{2}-\left(\frac{u_{c o}}{a_{c o}}\right)^{2}}=\sqrt{\left(\frac{2 \pi}{\lambda} n_{c o}\right)^{2}-\left(\frac{u_{c o}}{a_{c o}}\right)^{2}}
$$

where $\mathrm{k}$ is the free space propagation constant. Using (6), we obtain the effective refractive index of the core as follows [17]:

$$
n_{e f f, c o}=\frac{\beta_{c o}}{k}=\frac{\beta_{c o} \lambda}{2 \pi}
$$

Considering the set of fiber parameters shown in Table $\mathrm{I}$, the value $\mathrm{n}_{\text {eff,co }}=1.4523316$ is obtained using the software OptiGrating for the fundamental mode $\left(L P_{01}\right)$. The value found for the core effective refractive index lies between $n_{\text {clad }}<n_{\text {eff,co }}<n_{c o}$, and also matches with the one obtained by solving (3) for the $\mathrm{LP}_{01}$ mode employing the graphical method, shown in Fig.1.

TABLE I. PARAMETERS IN SIMULATION MODEL

\begin{tabular}{ccc}
\hline Symbol & Parameter & Value \\
\hline $\mathrm{a}_{\mathrm{co}}$ & Core radius & $2.5 \mu \mathrm{m}$ \\
$\mathrm{a}_{\text {clad }}$ & Cladding radius & $62.5 \mu \mathrm{m}$ \\
$\mathrm{n}_{\mathrm{co}}$ & Core refractive index & 1.458 \\
$\mathrm{n}_{\text {clad }}$ & Cladding refractive index & 1.45 \\
$\mathrm{n}_{\text {ext }}$ & External refractive index & 1 \\
$\Lambda$ & Central wavelength & $1450 \mathrm{~nm}$ \\
\hline
\end{tabular}




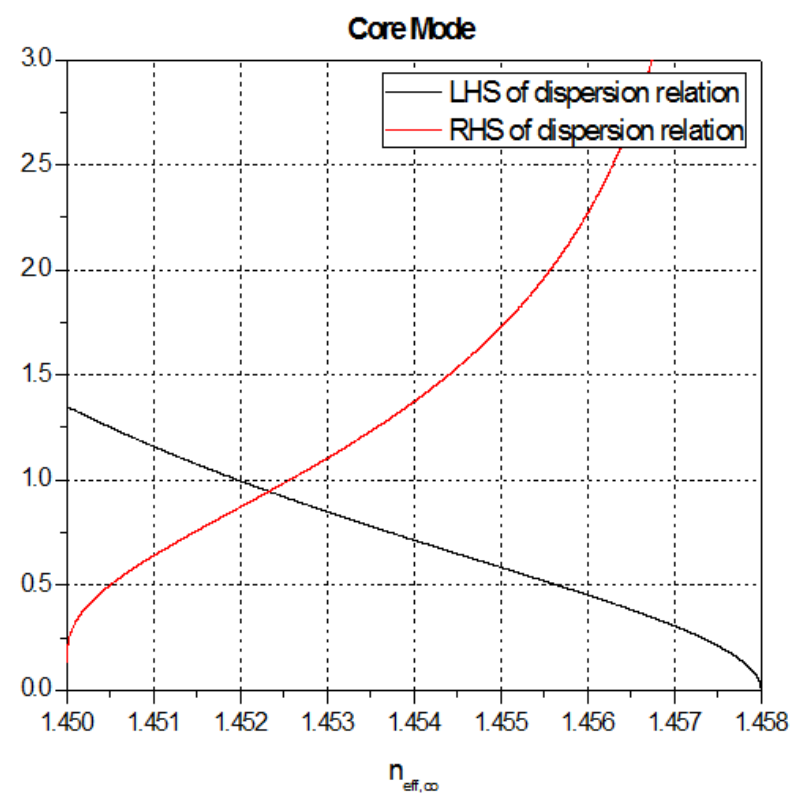

Fig 1. Solution by the graphical method pointing towards the intersection of the two functions present in the dispersion relation for the fundamental mode of the core.

\section{DETERMINING THE EFFECTIVE REFRACTIVE INDICES OF THE CLADDING - TWO LAYER GEOMETRY}

The Two-Layer Geometry approach allows the calculation of the effective refractive indices of the cladding ignoring the presence of the core [18]. So, this method is very similar to the procedure for determining the core effective refractive index, because the fiber geometry is once again based on only two layers, which in this case are the cladding and surrounding medium [9, 19]. However, this approach presents a multimode step-index structure whose core and cladding acts as one multi-mode fiber and the surrounding environment as the new cladding in the LPG region [9]. This leads to a modification in the normalized frequency $(V)$ and normalized transverse wave numbers, given by:

$$
\begin{aligned}
& V=\left(\frac{2 \pi a_{\text {clad }}}{\lambda}\right) \sqrt{n_{\text {clad }}^{2}-n_{\text {ext }}^{2}} \\
& u_{\text {clad }}=\sqrt{V^{2}-w_{\text {clad }}^{2}}
\end{aligned}
$$

Thus, we use a graphical solution again and plot the left-hand and right-hand sides of dispersion relation for the cladding modes, given by:

$$
u_{\text {clad }}\left(\frac{J_{1}\left(u_{\text {clad }}\right)}{J_{0}\left(u_{\text {clad }}\right)}\right)=w_{\text {clad }}\left(\frac{K_{1}\left(w_{\text {clad }}\right)}{K_{0}\left(w_{\text {clad }}\right)}\right)
$$

Owing to the large diameter of the cladding, it is able to guide many different light modes. Thus, a graphical representation, seen in Figs. 2-3, will show many intersection points each one corresponding to the eigenvalue that satisfies (10), and specifies the normalized transverse wave numbers related to a specific cladding mode. Therefore, the propagation constant corresponding to each cladding mode of order $m,\left(\beta_{\text {clad }}^{(m)}\right)$, can be determined from the related $u_{\text {clad }}^{(m)}$ and $w_{\text {clad }}^{(m)}$, as follows: 


$$
\beta_{\text {clad }}^{(m)}=\sqrt{\left(k n_{\text {clad }}\right)^{2}-\left(\frac{u_{\text {clad }}^{(m)}}{a_{\text {clad }}}\right)^{2}}=\sqrt{\left(\frac{2 \pi}{\lambda} n_{\text {clad }}\right)^{2}-\left(\frac{u_{\text {clad }}^{(m)}}{a_{\text {clad }}}\right)^{2}}
$$

Once we have calculated the propagation constants of the cladding modes, it is possible to determine the effective refractive indices using:

$$
n_{\text {eff }, \text { clad }}^{(m)}=\frac{\beta_{\text {clad }}^{(m)}}{k}=\frac{\beta_{\text {clad }}^{(m)} \lambda}{2 \pi}
$$

\section{DETERMINING THE EFFECTIVE REFRACTIVE INDICES OF THE CLADDING - THREE LAYER GEOMETRY}

This method proposes a more accurate description of mode propagation in the cladding. For this approach the core presence is not ignored any longer. This means that the cladding modes are calculated considering three layers, which in this case are the core, cladding and external medium. Thus, it permits us to obtain the exact value of the cladding modes corresponding to the three-layer fiber geometry. The expression for the dispersion relation for a cladding mode is given by [10-11]:

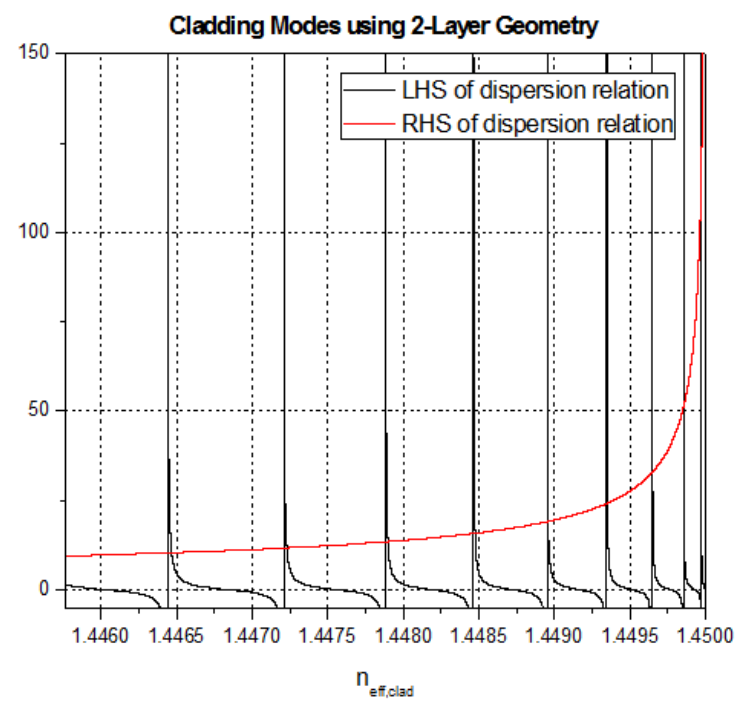

Fig. 2. Solution by the graphical method representing the dispersion relation condition for the $\mathrm{LP}_{0 \mathrm{~m}}$ cladding modes using the Two-Layer model. Intersecting points on default MATLAB vertical lines must be ignored. 


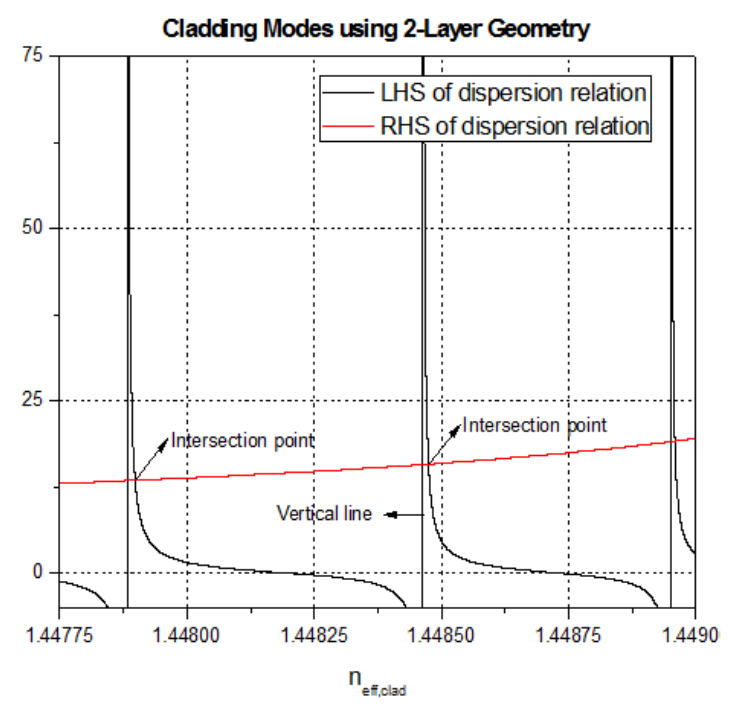

Fig. 3. Details of the intersection between the two functions of dispersion relation for some cladding modes. By default, vertical asymptotes are drawn as solid vertical lines, which are ignored for intersection points.

$$
\xi_{0}=\xi_{0}^{\prime}
$$

where [10-11]:

$$
\begin{aligned}
& \xi_{0}=\frac{1}{\sigma_{2}} \frac{u_{2}\left(J K+\frac{\sigma_{1} \sigma_{2} u_{21} u_{32}}{n_{\text {clad }}^{2} a_{c o} a_{c l a d}}\right) p_{l}\left(a_{\text {clad }}\right)-K q_{l}\left(a_{c l a d}\right)+J r_{l}\left(a_{c l a d}\right)-\frac{1}{u_{2}} s_{l}\left(a_{c l a d}\right)}{-u_{2}\left(\frac{u_{32}}{n_{c l a d}^{2} a_{c l a d}} J-\frac{u_{21}}{n_{c o}^{2} a_{c o}} K\right) p_{l}\left(a_{c l a d}\right)+\frac{u_{32}}{n_{c o}^{2} a_{c l a d}} q_{l}\left(a_{c l a d}\right)+\frac{u_{21}}{n_{c o}^{2} a_{c o}} r_{l}\left(a_{c l a d}\right)} \\
& \xi_{0}^{\prime}=\sigma_{1} \frac{u_{2}\left(\frac{u_{32}}{a_{\text {clad }}} J-\frac{n_{\text {ext }}^{2} u_{21}}{n_{\text {clad }}^{2} a_{c o}} K\right) p_{l}\left(a_{\text {clad }}\right)-\frac{u_{32}}{a_{\text {clad }}} q_{l}\left(a_{c l a d}\right)-\frac{u_{21}}{a_{c o}} r_{l}\left(a_{c l a d}\right)}{u_{2}\left(\frac{n_{\text {ext }}^{2}}{n_{\text {clad }}^{2}} J K+\frac{\sigma_{1} \sigma_{2} u_{21} u_{32}}{n_{c o}^{2} a_{c o} a_{c l a d}}\right) p_{l}\left(a_{c l a d}\right)-\frac{n_{\text {ext }}^{2}}{n_{c o}^{2}} K q_{l}\left(a_{c l a d}\right)+J r_{l}\left(a_{c l a d}\right)-\frac{n_{c l a d}^{2}}{n_{c o}^{2} u_{2}} s_{l}\left(a_{c l a d}\right)}
\end{aligned}
$$

The following definitions have been used in (14-16):

$$
\begin{aligned}
& \sigma_{1}=\frac{i l n_{\text {eff }}}{Z_{0}} \\
& \sigma_{2}=i \ln _{\text {eff }} Z_{0} \\
& u_{1}=\frac{2 \pi}{\lambda} \sqrt{\left(n_{\text {co }}^{2}-n_{\text {eff }}^{2}\right)} \\
& u_{2}=\frac{2 \pi}{\lambda} \sqrt{\left(n_{\text {clad }}^{2}-n_{\text {eff }}^{2}\right)} \\
& w_{3}=\frac{2 \pi}{\lambda} \sqrt{\left(n_{\text {eff }}^{2}-n_{\text {ext }}^{2}\right)} \\
& u_{21}=\frac{1}{u_{2}^{2}}-\frac{1}{u_{1}^{2}} \\
& u_{32}=\frac{1}{w_{3}^{2}}+\frac{1}{u_{2}^{2}}
\end{aligned}
$$


$J=\frac{1}{2}\left(\frac{J_{l-1}\left(u_{1} a_{c o}\right)-J_{l+1}\left(u_{1} a_{c o}\right)}{u_{1} J_{l}\left(u_{1} a_{c o}\right)}\right)$

$K=-\frac{1}{2}\left(\frac{K_{l-1}\left(w_{3} a_{c l a d}\right)+K_{l+1}\left(w_{3} a_{c l a d}\right)}{w_{3} K_{l}\left(w_{3} a_{c l a d}\right)}\right)$

$p_{l}(r)=J_{l}\left(u_{2} r\right) N_{l}\left(u_{2} a_{c o}\right)-J_{l}\left(u_{2} a_{c o}\right) N_{l}\left(u_{2} r\right)$

$q_{l}(r)=\frac{1}{2}\left\{J_{l}\left(u_{2} r\right)\left[N_{l-1}\left(u_{2} a_{c o}\right)-N_{l+1}\left(u_{2} a_{c o}\right)\right]-N_{l}\left(u_{2} r\right)\left[J_{l-1}\left(u_{2} a_{c o}\right)-J_{l+1}\left(u_{2} a_{c o}\right)\right]\right\}$

$r_{l}(r)=\frac{1}{2}\left\{N_{l}\left(u_{2} a_{c o}\right)\left[J_{l-1}\left(u_{2} r\right)-J_{l+1}\left(u_{2} r\right)\right]-J_{l}\left(u_{2} a_{c o}\right)\left[N_{l-1}\left(u_{2} r\right)-N_{l+1}\left(u_{2} r\right)\right]\right\}$

$s_{l}(r)=\frac{1}{4}\left\{\left[J_{l-1}\left(u_{2} r\right)-J_{l+1}\left(u_{2} r\right)\right]\left[N_{l-1}\left(u_{2} a_{c o}\right)-N_{l+1}\left(u_{2} a_{c o}\right)\right]-\left[J_{l-1}\left(u_{2} a_{c o}\right)-J_{l+1}\left(u_{2} a_{c o}\right)\right]\left[N_{l-1}\left(u_{2} r\right)-\right.\right.$ $N l+1 u 2 r$

In (13-28), $Z_{0}=\sqrt{\mu_{0} / \epsilon_{0}}=377 \Omega$ is the electromagnetic impedance in vacuum, $\mathrm{N}$ is the Bessel function of the second kind, or the Neumann function. The dispersion relation given by (13-28) is straightforward to solve numerically. Also, our analysis is limited to LPGs with azimuthal order $1=1$, this means that the coupling occurs between the fundamental guided mode and the circularly symmetric cladding modes [10-11].

Thus, using (13), a graphical solution method allows direct calculation of the effective refractive indices of the cladding modes by determining the intersection points (excluding intersecting points on vertical lines) in the graphical representation of the dispersion relation, shown in Figs. 4-5.

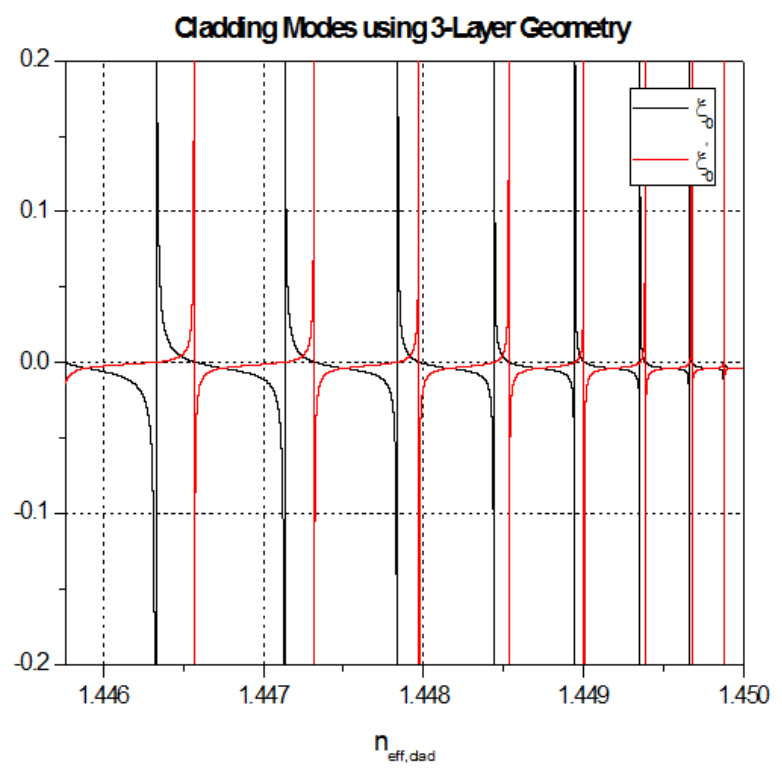

Fig. 4. Solution by the graphical method representing the dispersion relation condition for the LP0m cladding modes using the Three-Layer model. Intersecting points on default MATLAB vertical lines must be ignored. 


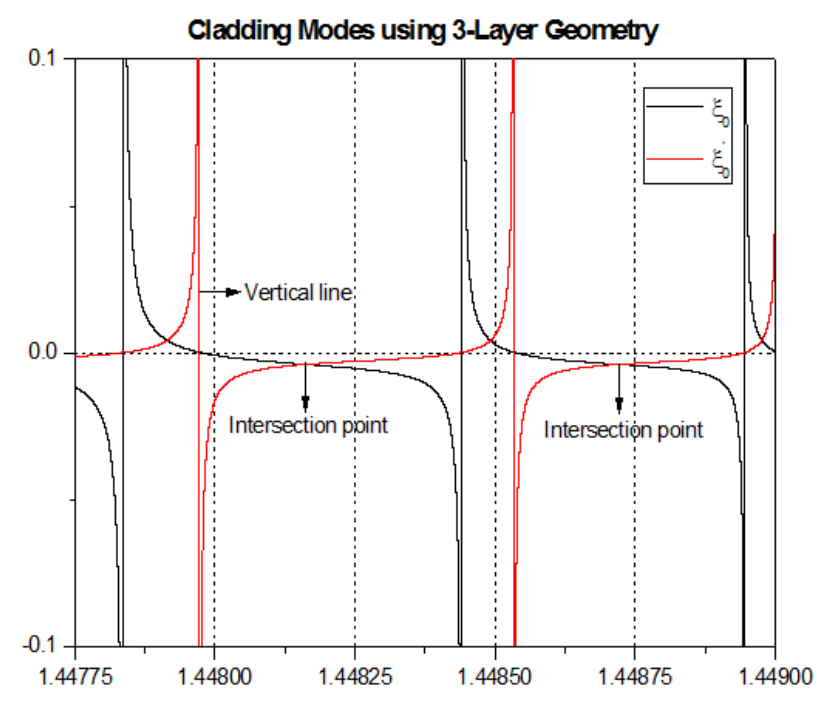

Fig. 5. Details of the intersection between the two functions of dispersion relation for some cladding modes. By default, vertical asymptotes are drawn as solid vertical lines, which are ignored for intersection points.

It is important to note that the vertical lines of the graphical representation, shown in Figs. 2-5, are not really part of the function. This means that the intersecting points on these asymptotes must be neglected, the vertical asymptotes only indicate that the distance between the curve and the line approaches zero as they tend to infinity.

\section{COMPARISON BETWEEN THE TWO-LAYER AND THREE-LAYER GEOMETRIES}

Distinguishing from the Two-Layer model, which graphically obtains the intersection points from a set of normalized transverse wave numbers, the Three-Layer model uses a range of cladding refractive indices to find the intersection points. The values found for the cladding effective refractive indices using both Two-Layer and Three-Layer geometries lie between $n_{\text {ext }}<n_{\text {eff,clad }}^{(m)}<n_{\text {clad }}$, and were obtained using the same parameters shown in Table I.

The set of parameters shown in Table I were also used to calculate the values of the effective refractive indices of the core and cladding modes using the software the OptiGrating. Table II summarizes a comparison among all the results of the effective indices of some LP modes at $\lambda=1.45$ $\mu \mathrm{m}$.

We note that the effective indices of different cladding modes present a drastic difference for both geometries evaluated. On the other hand, the effective refractive index of the fundamental mode of the core $\left(L P_{01}\right)$ is equal to both geometries and OptiGrating software, as expected since they use the weak guided mode theory $[8,11]$.

Furthermore, as observed in Table II, the Three-Layer model calculated values are in accordance with those obtained by the Optigrating, so that it is possible to validate the high accuracy of the ThreeLayer model of effective refractive indices calculation. 
TABLE II. COMPARISON OF THE EFFECTIVE REFRACTIVE INDICES OF THE CORE AND CLADDING MODES VALUES CALCULATED BY THE Two-LAYER AND THREe-LAYER GeOMETRIES AND OPTIGRATING V.2.2.

\begin{tabular}{lccc}
\hline \multirow{2}{*}{ Mode } & \multicolumn{3}{c}{ Effective Index } \\
\cline { 2 - 4 } & 2-Layer Geometry & 3-Layer Geometry & OptiGrating \\
\hline $\boldsymbol{L} \boldsymbol{P}_{\mathbf{0 1}}$ & 1.452332 & 1.452332 & 1.4523316 \\
$\mathbf{L} \boldsymbol{P}_{\mathbf{0 2}}$ & 1.449858 & 1.449954 & 1.4499581 \\
$\mathbf{L} \boldsymbol{P}_{\mathbf{0 3}}$ & 1.449650 & 1.449801 & 1.4498174 \\
$\mathbf{L} \boldsymbol{P}_{\mathbf{0 4}}$ & 1.449351 & 1.449543 & 1.4495803 \\
$\boldsymbol{L} \boldsymbol{P}_{\mathbf{0 5}}$ & 1.448959 & 1.449182 & 1.4492489 \\
\hline
\end{tabular}

The difference in effective refractive index calculation between the two and three layer model can be significant in sensing applications due to the resolution measurement that relies in resonant wavelength shifts with magnitude of some picometers. The refractive index error of $1 \%$ corresponds to an approximate $15 \mathrm{~nm}$ error in resonant wavelengths determination [20].

Figure 6 shows the experimental data used to verify the simulation results, collected using a $330 \mu \mathrm{m}$ period LPG, showing a resonant peak located at $1559 \mathrm{~nm}$. Figure 7 compares the experimental result with the Three-Layer Model and shows a difference in resonant peak wavelength of approximately 3 $\mathrm{nm}$, which can be relevant for sensing systems. The Two-Layer Model yields a higher displacement in the resonant peak wavelength due to higher error in the effective refractive index, as can be seen in Table II, therefore, should not be used in sensing applications.

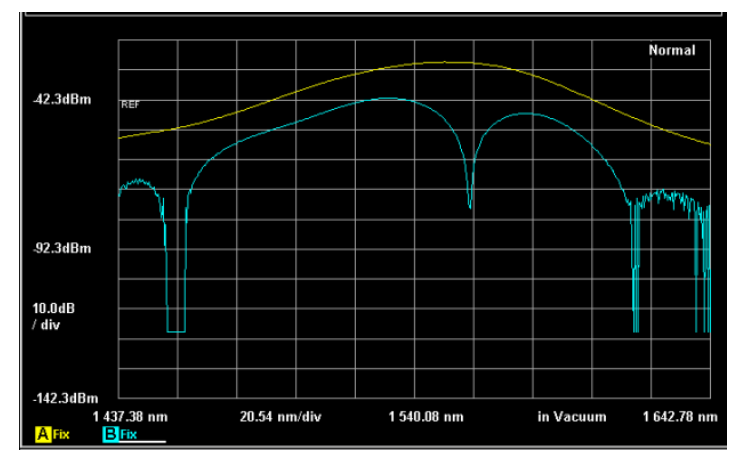

Fig. 6. Output of the Optical Spectrum Analyzer in response to a $330 \mu \mathrm{m}$ period LPG. 


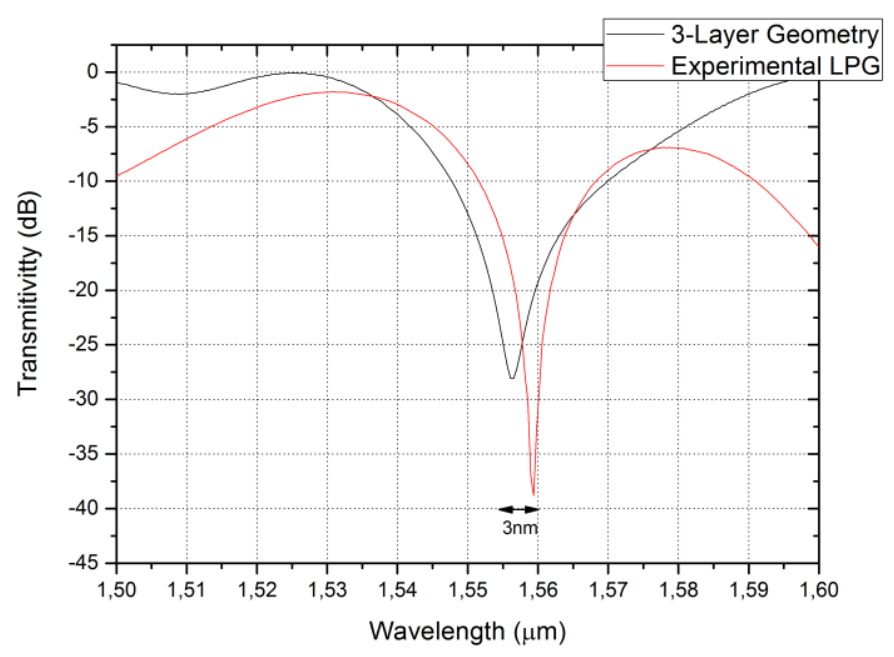

Fig. 7. Detail of the difference in resonant peak wavelength between experimental data and Three-Layer Model.

\section{CONCLUSION}

We reported a complete and detailed analysis of the propagation characteristics of the core and cladding modes of the LPG. The cladding analysis showed distinct results for the effective refractive indices in both geometries evaluated. In addition, the results obtained by the Three-Layer geometry were validated with the OptiGrating and experimentally. Thus, a complete analysis of the Three-Layer model is required to model the cladding modes in LPGs. On the other hand, the simple Two-Layer approach may lead to erroneous design of the LPGs characteristics.

\section{ACKNOWLEDGMENT}

The authors thank for the support provided by Propesq/UFJF and FAPEMIG.

\section{REFERENCES}

[1] S.F. Wang and C.C. Chiang, "A Notched Long-Period Fiber Grating Magnetic Field Sensor Based on Nanoparticle Magnetic Fluid," Applied Sciences, vol. 6, no. 1, p. 9, Jan. 2016.

[2] Coelho, Thiago V.N.; Pontes, M.Jose; Santos, Jose L.; dos Santos, A.Bessa; Silveira, Daniel D.; Silva, Vinicius N.H.; Lopez -Barbero, A.P.; Delgado, Felipe S.; Neto, Presley X., "A numerical and experimental study of the remote long-period grating fiber sensor with Raman Amplification," in Microwave and Optoelectronics Conference (IMOC), 2015 SBMO/IEEE MTTS International, vol., no., pp.1-6, 3-6 Nov. 2015

[3] Askarov, D.; Kahn, J.M., "Long-Period Fiber Gratings for Mode Coupling in Mode-DivisionMultiplexing Systems," in Lightwave Technology, Journal of , vol.33, no.19, pp.4032-4038, Oct.1, 12015

[4] Delgado, F.S.; Silveira, D.D.; Coelho, T.V.N.; Bessa dos Santos, A., "Mathematical modelling for correlation between temperature and mechanical strain in long period gratings," in SENSORS, 2014 IEEE , vol., no., pp.1900-1903, 2-5 Nov. 2014

[5] A. Vengsarkar, N. Bergano, C. Davidson, J. Pedrazzani, J. Judkins, and P. Lemaire, "Long-period fiber-grating-based gain equalizers," Opt. Lett. 21, 336-338 (1996). 
[6] Biswas, P.; Basumallick, N.; Bandyopadhyay, S.; Dasgupta, K.; Ghosh, A.; Bandyopadhyay, S., "Sensitivity Enhancement of Turn-Around-Point Long Period Gratings By Tuning Initial Coupling Condition," in Sensors Journal, IEEE, vol.15, no.2, pp.1240-1245, Feb. 2015

[7] Qin Chen; Lee, J.; Minren Lin; Yong Wang; Yin, Stuart Shizhuo; Qiming Zhang; Reichard, K.M., "Investigation of tuning characteristics of electrically tunable long-period gratings with a precise four-Layer model," inLightwave Technology, Journal of , vol.24, no.7, pp.2954-2962, July 2006

[8] D. Gloge, "Weakly Guiding Fibers," Appl. Opt. 10, 2252-2258 (1971).

[9] Vengsarkar, A.M.; Lemaire, P.J.; Judkins, J.B.; Bhatia, V.; Erdogan, T.; Sipe, J.E., "Long-period fiber gratings as band-rejection filters," in Lightwave Technology, Journal of , vol.14, no.1, pp.5865, Jan 1996.

[10] T. Erdogan, "Cladding-mode resonances in short- and long-period fiber grating filters," J. Opt. Soc. Am. A 14, 1760-1773 (1997).

[11] T. Erdogan, "Cladding-mode resonances in short- and long-period fiber grating filters: errata," J. Opt. Soc. Am. A 17, 2113-2113 (2000).

[12] E. Anemogiannis, E. N. Glytsis, and T. K. Gaylord, "Transmission characteristics of long period fiber gratings having arbitrary azimuthal/radial refractive index variations", J. Lightwave Technol., vol. 21, no. 1, pp. 218227,Jan. 2003"

[13] J. A. Buck, Fundamentals of optical fiber, $2^{\text {nd }}$ ed., Wiley series of Pure and Applied Optics, 2004.

[14] G. Keiser, Optical Fiber Communications, $4^{\text {th }}$ ed., McGraw-Hill, 2010.

[15] L. H. Binh, Optical Fiber Communications Systems: Theory and Practice with MATLAB and Simulink Models, $1^{\text {st }}$ ed., CRC Press, 2010.

[16] W. B. Jr. Jones, Introduction to Optical Fiber Communication Systems, New York: Holt, Rinehart \& Winston, 1988.

[17] C. J. E. S. Mendes, Estruturas sensoras em fibra óptica para monitorização ambiental baseado em redes de período longo, in Departamento de Física. Faculdade de sensoras, Monitorização de Porto: Porto, 2007.

[18] H. Patrick, A. Kersey, and F. Bucholtz, "Analysis of the Response of Long Period Fiber Gratings to External Index of Refraction," J. Lightwave Technol. 16, 1606- (1998).

[19] S. W. James and R. P. Tatam, "Optical fibre long-period grating sensors: Characteristics and application," Meas. Sci. Technol. 14, R49-R61 (2003).

[20] A. Arabi and L. L. Goddard, "Measurements of the refractive indices and thermo-optic coefficients of $\mathrm{Si}_{3} \mathrm{~N}_{4}$ and $\mathrm{SiO}_{x}$ using microring resonances", Optics Letters, vol. 38, no. 19, October, 2013. 